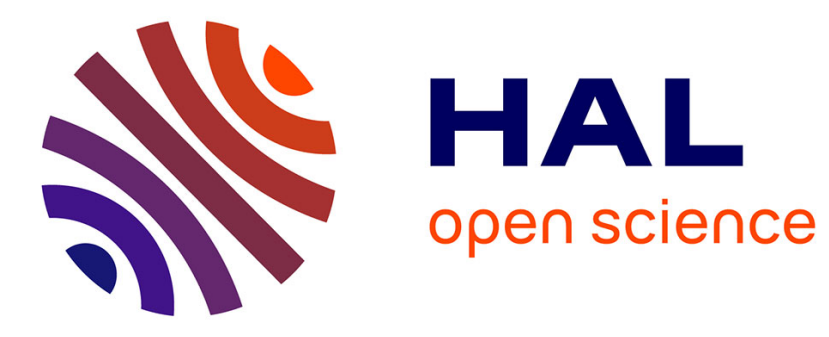

\title{
Probing keto-enol tautomerism using photoelectron spectroscopy
}

Nathalie Capron, Bastien Casier, Nicolas Sisourat, Maria Novella Piancastelli, Marc Simon, Stéphane Carniato

\section{- To cite this version:}

Nathalie Capron, Bastien Casier, Nicolas Sisourat, Maria Novella Piancastelli, Marc Simon, et al.. Probing keto-enol tautomerism using photoelectron spectroscopy. ChemPhysChem, 2015, 17 (30), pp.19991-19996. 10.1039/C5CP02023A . hal-01198819

\section{HAL Id: hal-01198819 https://hal.sorbonne-universite.fr/hal-01198819}

Submitted on 14 Sep 2015

HAL is a multi-disciplinary open access archive for the deposit and dissemination of scientific research documents, whether they are published or not. The documents may come from teaching and research institutions in France or abroad, or from public or private research centers.
L'archive ouverte pluridisciplinaire HAL, est destinée au dépôt et à la diffusion de documents scientifiques de niveau recherche, publiés ou non, émanant des établissements d'enseignement et de recherche français ou étrangers, des laboratoires publics ou privés. 


\title{
Probing keto-enol tautomerism with photoelectron spectroscopy.
}

\author{
Nathalie Capron, ${ }^{1,2}$ Bastien Casier, ${ }^{1,2}$ Nicolas Sisourat, ${ }^{1,2}$ Maria \\ Novella Piancastelli, ${ }^{1,2,3}$ Marc Simon,, ${ }^{1,2}$ and Stéphane Carniato ${ }^{1,2, *}$ \\ ${ }^{1}$ Sorbonne Universités, UPMC Univ Paris 06, UMR 7614, \\ Laboratoire de Chimie Physique Matière et Rayonnement, F-75005, Paris, France \\ ${ }^{2}$ CNRS, UMR 7614, Laboratoire de Chimie Physique \\ Matière et Rayonnement, F-75005, Paris, France \\ ${ }^{3}$ Department of Physics and Astronomy, \\ Uppsala University, 75120 Uppsala, Sweden
}

(Dated: September 14, 2015)

\begin{abstract}
We theoretically investigate the mechanism of tautomerism in the gas-phase acetylacetone molecule. The minimum energy path between the enolone and diketo forms has been computed using the Nudged-Elastic Band (NEB) method within the density-functional theory (DFT) using the projector augmented-wave method and generalized gradient approximation in Perdew-Wang (PW91) parametrization. The lowest transition state as well as several intermediate geometries between the two stable tautomers have been identified. The outer-valence ionization spectrum for all determined geometries has been computed using the third-order non-Dyson algebraic diagrammatic construction technique. Furthermore, the oxygen core-shell ionization spectrum for these geometries has been obtained with DFT and the Becke three-parameter Lee-Yang-Parr (B3LYP) functional. It is shown that all spectra depend strongly on the geometries demonstrating the possibility to follow the proton-transfer dynamics with photoelectron spectroscopy in pump-probe experiments.
\end{abstract}

\footnotetext{
* Nicolas.Sisourat@upmc.fr
} 


\section{INTRODUCTION}

Photoelectron spectroscopy is a powerful tool to study the electronic structure of occupied core and valence levels of isolated species $^{1-3}$. With the recent advances in ultrashort and bright XUV and X-ray sources, femtosecond time-resolved photoelectron spectroscopy is now possible, which allows to follow the dynamics of a chemical reaction on the relevant timescale $^{4}$. Most of the studies have focused on excited-state dynamics since pump intense femtosecond laser pulses in the corresponding spectral region are experimentally more accessible. However, the continuous progresses in intense and femtosecond mid-infrared laser technology ${ }^{8-11}$ open the way to direct studies of photo-induced chemical reactions within the ground state $\mathrm{s}^{12-15}$. One should also mention that vibrational excitation of molecules in ground electronic state can be achieved with near-infrared lasers by adiabatic passage techniques $^{5-7}$

Among the class of chemical reactions, proton-transfer processes are of prime importance in chemistry and biochemistry ( ${ }^{16-18}$ and references therein). Keto-enol tautomerism is a prototype of such processes which has been, and is still, widely investigated. Tautomerism in $\beta$-diketone compounds is a particular case since the enolone (also called keto-enol) form of most $\beta$-diketones is more stable than the diketo form owing to strong intramolecular hydrogen bonds. The structure and reactivity of such molecules have attracted considerable attention $^{19-26}$.

In this article we investigate the mechanism of keto-enol tautomerism in the gas-phase acetylacetone (Pentane-2,4-dione) molecule. The latter is one of the simplest $\beta$-diketones. Stability of the different forms in gas-phase and in different solvents have been extensively investigated $^{19-21,27-39}$. The situation is different for the interconversion mechanism. While the tautomeric mechanism in liquid water in which solvent molecules participate to the proton transfer is commonly accepted ${ }^{39}$, the mechanism in gas-phase has been less studied ${ }^{19,21}$. Furthermore, we demonstrate that the outer-valence and the oxygen core shell ionization spectra of the acetylacetone molecule can be used to probe and follow the dynamics of the proton transfer. It should be mentioned that photoelectron spectroscopy has been already used to identify the enolone and diketo forms of this molecule at different temperatures ${ }^{40}$. We show here that the transition state as well as intermediate geometries between the two stable tautomers can also be traced by inner-shell and valence electron spectroscopies. 
The outline of the article is the following: in section II, we present the numerical methods used to determine the dynamics of proton transfer in acetylacetone and employed to compute the corresponding ionization spectra. In section III.A, the geometries of the two tautomers obtained in this work are discussed with respect to previous studies. The geometry and energy of the transition and intermediate states are then reported in section III.B. Finally, the valence and core ionization spectra of the acetylacetone in all these geometries are presented and discussed in section III.C.

\section{COMPUTATIONAL DETAILS}

We have modeled the tautomerism dynamics in the acetylacetone molecule and have simulated the ionization spectra of the latter during this process. It is assumed that the system follows the minimum energy path between the two tautomeric forms. This path was computed using the Nudged-Elastic Band (NEB) method ${ }^{41}$ as implemented in VASP ${ }^{42,43}$. We used density-functional theory (DFT) with the projector augmented-wave (PAW) method ${ }^{44}$ and generalized gradient approximation (GGA) in Perdew-Wang (PW91) parametrization ${ }^{45}$. As we consider an isolated molecule inside the unit cell, only the Gamma-point is used inside the reciprocal unit cell. It should also be mentionned that the VASP relies on the use of plane-wave basis set which is less suitable than, for example a Gaussian basis set, to describe isolated species. However, convergence of the results with respect to the box size has been checked. Moreover, the present method can readily be applied to molecules embedded in chemical environment, like in a solvent for example, for which the plane-wave basis set is advantageous. In total 8 intermediate geometries between the diketo and the enolone forms have been obtained. It should be noted that thermal corrections are neglected here and only electronic energies are given. As pointed out in Ref. ${ }^{19}$, at room temperature these corrections change the energy barrier by about $5 \%$ only.

The outer-valence ionization spectra of the acetylacetone molecule in these geometries as well as in the diketo and the enolone forms have been computed with a home-made code based on the so-called algebraic diagrammatic construction technique at the third order $(\mathrm{ADC}(3))$ within the non-Dyson approach $^{46,47}$ (see also Ref. ${ }^{48}$ for a recent review on ADC methods). This approach provides accurate outer and inner-valence ionization energies: typical absolute errors are expected to be around $0.2-0.3 \mathrm{eV}^{47,48}$. The Hartree- 
Fock (HF) self-consistent-field (SCF) molecular orbitals and the integrals needed for the ADC calculations were computed using the GAMESS(US) ab-initio program ${ }^{49}$. A 6-311G basis set was used ${ }^{50}$. In the ADC calculations we kept the 7 core molecular orbitals frozen and limited the active space to the first 88 orbitals. Convergence of the results with respect to the basis set and the active space has been checked. All spectra have been shifted by $+0.25 \mathrm{eV}$ such that the lowest peak agrees with the experimental data of the diketo and the enolone forms ${ }^{51}$. Furthermore, the spectra have been convoluted with a gaussian function with $0.3 \mathrm{eV}$ full width at half maximum in order to take into account the rovibrational broadening and typical current experimental resolution.

At the third order, the ADC method reproduces less accurately the binding energy of the core-electron since relaxation energy, which is expected to be large from the neutral to the core-ionized states, is only partially taken into account ${ }^{52}$. The ionization spectra for the K-shell electron of the oxygen atoms have been computed at the SCF/DFT level of theory, using the GAMESS(US) package ${ }^{49}$. The relaxation energy is thus accounted for by using the SCF approximation, which consists of a closed-shell SCF calculation for the neutral molecule and a restricted open-shell HF calculation (ROHF) for the ionized core states. The basis sets used for carbon and hydrogen atoms for the neutral states are of 10s4p1d 6-31G*53 type, in a [3s,2p,1d] contraction. For the oxygen atoms, the 6-31G basis set is augmented by a set of three polarization d functions and a (3s,3p) set of diffuse basis functions in order to treat properly the hydrogen bond in the enolone form. For the core hole calculations, we used the basis set (denoted as O-631G, see appendix) optimized by Carniato and Millié ${ }^{54}$ augmented by a set of three polarization d functions and a (3s,3p) set of diffuse basis functions in order to treat properly the hydrogen bond and charge transfer in core ionized states. The DFT calculations were carried out at the gradient-corrected DFT level using the Becke three-parameter exchange functional ${ }^{55}$ along with the Lee-Yang-Parr ${ }^{56}$ gradient-corrected correlation functional, the so-called Becke three-parameter Lee-Yang-Parr (B3LYP) functional. The combined use of this optimized basis set and the Kohn-Sham technique allows to treat large molecular systems and has proven to provide binding energies with only a few tenths of an electron volt deviations from experimental data ${ }^{54}$. Relativistic effects $(0.4 \mathrm{eV})$ on the core-binding energy of oxygen 1s have been considered ${ }^{57}$. 


\section{RESULTS AND DISCUSSION}

\section{A. Geometry of the enolone and diketo form}

The computed geometry and energy of the enolone and diketo forms obtained at the PAW/GGA level with a plane-wave basis set are compared to previous studies. Selected geometrical parameters are compared in Table I and Table II for the enolone and diketo forms, respectively. The agreement between the data is satisfactory. The most noticeable difference is the value of dihedral angle between the planes of the carbonyls for the diketo form. These two conformers are close in energy such that the energy difference between them is below the accuracy of our numerical approach, which explains the difference in the value of dihedral angle.

The energy difference between the two tautomeric forms $\left(\mathrm{E}_{\text {diketo }}-\mathrm{E}_{\text {enolone }}\right)$ is reported in Table III. Our calculations predict a sligthly larger energy difference compared to Belova et $a l .{ }^{20}$ and Sliznev et $a l .{ }^{26}$. The difference can be attributed to the use of different functionals. As shown below, this energy difference is small compared to the energy of the transition state. The effects of the different functionals are therefore negligeable for this study. Furthermore, the geometrical parameters, which are the important input data for determining the ionization spectra, compare well with other calculations.

\begin{tabular}{|c|c|c|c|}
\hline & Tayyari et al. $^{23}$ & ${\text { Belova } \text { et } \text { al. }^{20,38}}$ & present work \\
\hline $\mathrm{d}_{O_{2}-H_{5}}(\AA)$ & 1.00 & 1.00 & 1.06 \\
\hline $\mathrm{d}_{C_{2}-O_{1}}(\AA)$ & 1.26 & 1.25 & 1.27 \\
\hline $\mathrm{d}_{C_{4}-O_{2}}(\AA)$ & 1.34 & 1.32 & 1.32 \\
\hline $\mathrm{d}_{C_{3}-C_{4}}(\AA)$ & 1.37 & 1.37 & 1.38 \\
\hline $\mathrm{d}_{C_{3}-C_{2}}(\AA)$ & 1.44 & 1.44 & 1.43 \\
\hline $\mathrm{d}_{O_{1}-O_{2}}(\AA)$ & 2.54 & 2.53 & 2.47 \\
\hline$\alpha_{O_{1} H_{5} O_{2}}$ & $150.2^{\circ}$ & $149.13^{\circ}$ & $154.3^{\circ}$ \\
\hline
\end{tabular}

Table I. Selected geometrical parameters for the acetylacetone in the enolone form. See Fig. 1 for the labelling of the atoms. 


\begin{tabular}{|l|c|c|c|}
\hline & Belova et al. $^{20}$ & Sliznev et al. $^{26}$ & present work \\
\hline $\mathrm{d}_{C_{2}-O_{1}}(\AA)$ & 1.22 & 1.21 & 1.22 \\
\hline $\mathrm{d}_{C_{4}-O_{2}}(\AA)$ & 1.22 & 1.21 & 1.22 \\
\hline $\mathrm{d}_{C_{3}-C_{4}}(\AA)$ & 1.53 & 1.53 & 1.53 \\
\hline $\mathrm{d}_{C_{3}-C_{2}}(\AA)$ & 1.53 & 1.53 & 1.53 \\
\hline$\chi_{O_{1} C_{2} C_{3} C_{4}}$ & $89.1^{\circ}$ & $87.9^{\circ}$ & $105.8^{\circ}$ \\
\hline
\end{tabular}

Table II. Selected geometrical parameters for the acetylacetone in the diketo form. See Fig. 1 for the labelling of the atoms.

\begin{tabular}{|c|c|c|c|}
\hline & Belova et ll. $^{20}$ & Sliznev et al. ${ }^{26}$ & present work \\
\hline$\Delta \mathrm{E}(\mathrm{kcal} / \mathrm{mol})$ & 6.43 & 5.21 & 10.68 \\
\hline
\end{tabular}

Table III. Energy difference between the enolone and diketo forms.

\section{B. NEB results}

We now focus on the tautomeric mechanism which has been much less investigated than the geometries and energetics of the stable forms. Here, we assume that the molecule follows the minimum energy path. The latter has been obtained within the NEB calculations and its energetic profile is shown in Fig. 2. The energy of the diketo form is set to zero. As already shown in Table III, the enolone is more stable with an energy of $-0.46 \mathrm{eV}$ (10.68 $\mathrm{kcal} / \mathrm{mol})$. The transition state lies $2.61 \mathrm{eV}$ (60.09 kcal/mol) above the enolone form. The geometry of the enolone and diketo forms as well as of the transition state are shown in Fig. 1. Characteristic distances and angles for the three geometries are given in Tables IV and V. As major changes from the enolone geometry to the transition state one, the dihedral angle $\left(C_{3} C_{2} C_{4} O_{2}\right)$ rises to $70.20^{\circ}$ while the $\mathrm{O}_{2}-\mathrm{H}_{5}$ distance increases and both $\mathrm{CO}$ distances decrease as in a concerted mechanism. From the transition state to the diketo form the hydrogen of the alcohol function moves to the central carbon while the CO distances shorten to a characteristic double-bond value. The geometry of each form is given in Supporting Information.

In the following, we demonstrate that the minimum energy path between the two tautomeric forms can be determined by outer-valence and core ionization spectra, opening the possibility to experimentally follow the proton-transfer dynamics. However, the realization 


\begin{tabular}{|l|c|c|c|}
\hline & ${\text { Kaweetirawatt } \text { et }_{\text {al. }}{ }^{21}}$ & Alagona et al. $^{19}$ & present work \\
\hline $\mathrm{E}(\mathrm{kcal} / \mathrm{mol})$ & $\mathrm{NC}$ & 63.22 & 49.41 \\
\hline $\mathrm{d}_{O_{2}-H_{5}}(\AA)$ & 1.27 & 1.27 & 1.29 \\
\hline $\mathrm{d}_{C_{3}-H_{5}}(\AA)$ & 1.52 & 1.53 & 1.48 \\
\hline
\end{tabular}

Table IV. Selected geometrical parameters and energy (with respect to the diketo form) for the acetylacetone in the TS form. See Fig. 1 for the labelling of the atoms.

\begin{tabular}{|c|c|c|c|}
\hline & Enolone & TS & Diketo \\
\hline \hline $\mathrm{d}_{O_{7}-H_{9}}(\AA)$ & 1.06 & 1.29 & - \\
\hline $\mathrm{d}_{C_{3}-O_{7}}(\AA)$ & 1.32 & 1.29 & 1.22 \\
\hline $\mathrm{d}_{C_{2}-O_{6}}(\AA)$ & 1.27 & 1.23 & 1.22 \\
\hline$\chi_{O_{6} C_{2} C_{1} C_{3}}$ & $0.0^{\circ}$ & $70.2^{\circ}$ & $105.8^{\circ}$ \\
\hline
\end{tabular}

Table V. Selected geometrical parameters for the acetylacetone in the enolone, TS and diketo forms. See Fig. 1 for the labelling of the atoms.

of such experiments deserves a short discussion here. Owing to the high energy barrier, the enolone is the dominant form at room temperature and the diketo form exists only at higher temperature ${ }^{38}$. In order to follow the tautomeric dynamics, a pump-probe scheme in which the pump provides enough energy to the enolone form to surmount the barrier can be employed. The energy of the transition state can be reached by, for example, excitation of a given vibrational mode with an intense and short resonant mid-infrared laser. Other schemes are also possible ${ }^{5-7}$. Owing to intramolecular vibrational relaxation ${ }^{58}$, the excess energy dissipates over the entire molecule and may induce the tautomerism. The branching ratio of this path depends on the superposition of vibrational levels formed by the excitation pulse and thus on the latter. Furthermore, the system may not follow the minimum energy path if the excess energy largely exceeds the energy barrier. Accurate simulation of the response of such polyatomic molecule to a vibrational excitation is a challenging task and is beyond the present work. Here, we use the minimum energy path as one possible path which provides a realistic tautomeric mechanism. 


\section{Ionization spectra}

The outer-valence ionization spectrum of the enolone, diketo and the 8 NEB intermediate forms are shown in Fig. 3. The experimental data for the enolone and diketo forms obtained by Hush et al. ${ }^{51}$ are shown in the same figure. A good agreement for the two lowest peak is observed. For higher binding energies, the experimental resolution does not allow a fair comparison.

The lowest peaks, up to $14 \mathrm{eV}$, correspond successively to ionization of deeper orbitals (HOMO, (HOMO-1), ...). The density of the four outer most valence orbitals, which correspond to the peaks discussed below, is given for the enolone and diketo forms in Supporting Information. For higher binding energy, owing to the breakdown of the one-particle picture ${ }^{59}$ the states cannot be described as a single Hartree-Fock reference configuration with a hole in one molecular orbital. Instead configurations corresponding to two-holes within the occupied orbital space and one-particle in the virtual orbitals have substantial weights. In the following, we discuss only the region below $14 \mathrm{eV}$.

Comparing the spectra for the different NEB images, it is seen that the peak positions depend strongly on the form of the molecule: as the molecule changes from the enolone form to the geometry i5 (one before the transition state), the two lowest peaks are redshifted by up to $0.3 \mathrm{eV}$. These peaks for the transition state are close in energy to that of the enolone form, making the identification of the forms difficult. However, the two higher peaks are shifted with respect to each other for these two forms. It should be noted that the region between $11.5 \mathrm{eV}$ and $13 \mathrm{eV}$ is characteristic of each form. Between the transition state and the diketo form, a substantial increase (up to $1.3 \mathrm{eV}$ ) of the first two peaks is then observed. From i3 geometry to the diketo one, the peaks around 9-10 eV are not significantly affected. The next region (11.5-13 eV) is however influenced by the geometry.

The binding energy of the K-shell electron of the oxygen atoms for the enolone and diketo forms as well as for all intermediate geometries are shown in Fig. 4. The two oxygen atoms have a different chemical environment in the enolone form, they thus present two well-separated peaks which can be experimentally resolved. The oxygen in the enol function exhibits a peak at $538.6 \mathrm{eV}$ while that of the other oxygen atom is at $536.9 \mathrm{eV}$. This is in

good agreement with the experimental values (538.8 and $537.2 \mathrm{eV}$, respectively) ${ }^{60,61}$. The diketo form exhibits a single peak (the two oxygen atoms are equivalent) at $537.7 \mathrm{eV}$, in 
agreement with the experimental work of Perera et $a l .{ }^{62}$. The energy difference between the peaks for the geometries from the enolone form to the transition-state is larger than in the enolone geometry, reaching up to $3 \mathrm{eV}$. Between the transition-state form and the diketo one, the difference is less pronounced. Nevertheless, each geometry exhibits a characteristic spectrum which can be used to follow the tautomeric mechanism.

These results demonstrate that the outer-valence and core shell ionization spectra can be used to unambiguously identify the forms of the acetylacetone during the enolone tautomerism and thus to follow the proton-transfer dynamics.

\section{CONCLUSION}

We have investigated the tautomeric mechanism in gas-phase acetylacetone molecule. The lowest identified transition state lies $2.61 \mathrm{eV}$ above the enolone form. Most of this energy is employed to break the strong intramolecular bond by torsion of the carbon chain in a concerted mechanism. Such energy can, in principle, be reached by vibrational excitation using mid-infrared lasers which can thus be used to initiate the tautomerism process.

In total, 10 geometries along the minimum energy path have been determined. The outervalence and oxygen core-shell ionization spectra for each geometry have been computed. All spectra show a strong dependence on the geometry which opens the possibility to follow the proton-transfer dynamics in real-time using photoelectron spectroscopy. The comparison between experiment and theory, after oxygen $\mathrm{K}$ edge ionization or valence ionization, would allow to determine the tautomerism kinetics. In other words, the dynamics needed to go from one geometry to the next one would then be experimentally determined. In the view of the recent progresses in ultrashort and intense short wavelength sources as well as midinfrared laser technology, we hope the present theoretical work will encourage experimental development of mid-infrared pump - photoelectron probe spectroscopy.

\section{ACKNOWLEDGEMENTS}

We thank Professor Hafner (Faculty of Physics and Center for Computational Materials Science, University of Vienna, Austria) for allowing us to use the VASP code. 


\section{APPENDIX}

The basis set used for the calculations of the oxygen core-shell ionization spectra is detailed here. We briefly summarize how the basis set was computed.

Table VI. Set of coefficients and exponents corresponding to oxygen core ionized optimized unrestricted HartreeFock (UHF) configurations for 6-31G basis set as calculated in ${ }^{54}$

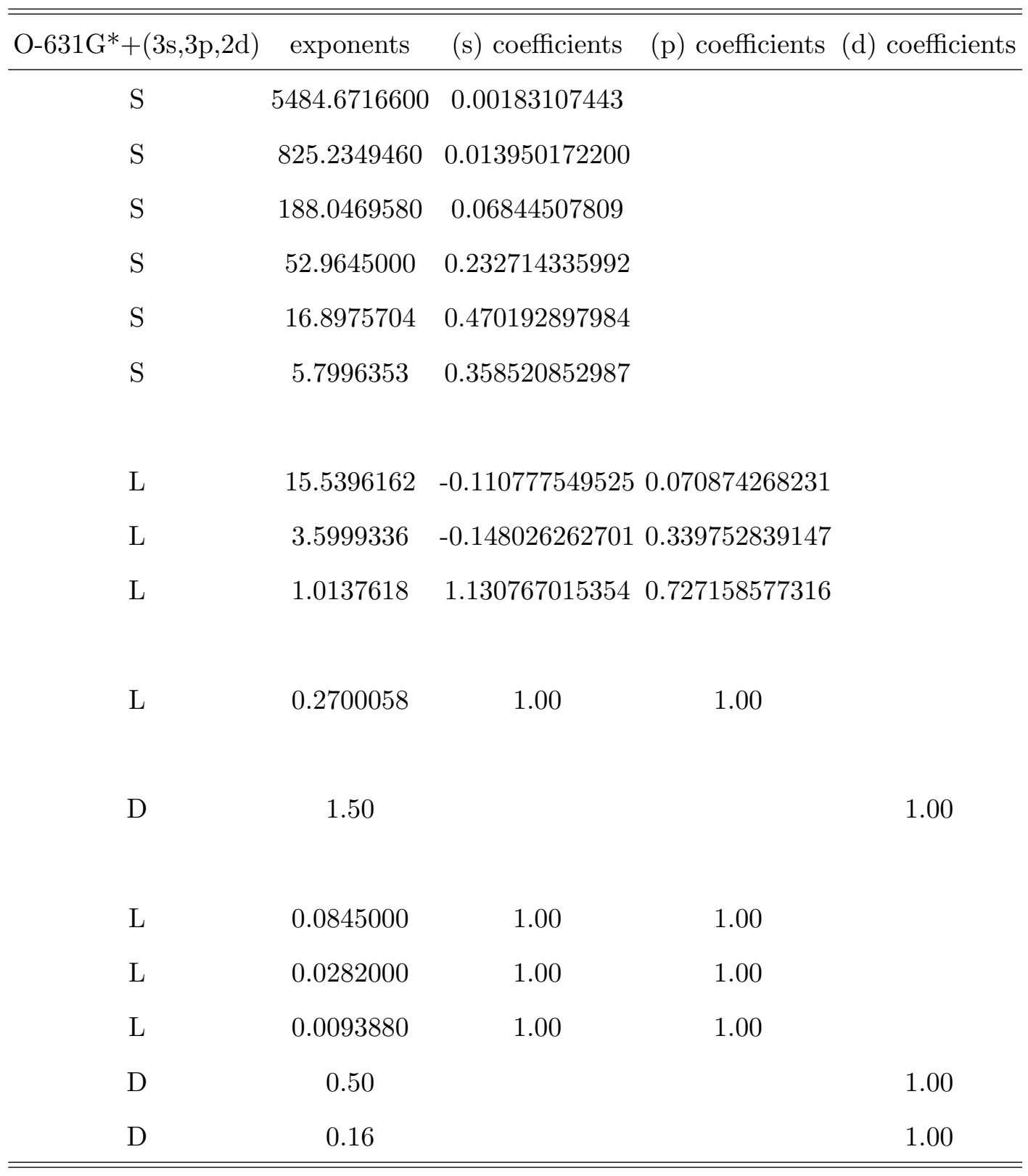

In order to obtain an improved representation of the relaxation effects in the core-ionized states, the basis set was obtained from HF calculations combined with a simulated annealing 
procedure where the parameters specifying the contraction coefficients and orbital exponents were determined by minimizing atomic SCF energies within the unrestricted HF scheme. The system is heated and thermalized at $10^{6} \mathrm{~K}$ during 500 steps (at each step, ten moves of both coefficients and exponents are individually proposed). After each ten steps, the configuration with lowest electronic energy ever encountered is saved. At the end of the thermalization, 50 selected configurations are then collected and ready to be cooled down slowly to $0 \mathrm{~K}$ following a simulated annealing approach. After each ten steps, the temperature is set to $\mathrm{T}_{\text {new }}=\mathrm{T}_{\text {old }}-\frac{T_{\text {old }}}{15}$. Near $0 \mathrm{~K}$, the set with the lowest electronic energies is retained and the simulated annealing procedure is stopped when the energy fluctuations are less than $10^{-10}$ atomic units. Furthermore, we added a set of three polarization d functions and a (3s,3p,2d) set of diffuse basis functions in order to treat properly the hydrogen bond and charge transfer in the core ionized state.

[1] K.Siegbahn, C.Nordling, G.Johansson, J.Hedman, P.F.Heden, K.Hamrin, U.Gelius, T.Bergmark, L.O.Werme, R.Manne and Y.Baer, ESCA applied to free molecules, North Holland, Amsterdam, London, 1969.

[2] D.W.Turner, A.D.Baker, C.Baker and C.R.Brundle, Molecular Photoelectron Spectroscopy, Wiley, New York, 1970.

[3] T.A.Carlson, Photoelectron and Auger spectroscopy, Plenum Press, New York, 1975.

[4] A. Stolow, A.E. Bragg and D.N. Neumark, Chem. Rev., 2004, 104, 1719.

[5] N.V Vitanov, T. Halfmann, B.W. Shore and K. Bergmann, Annu. Rev. Phys. Chem., 2011, $\mathbf{5 2 ,} 763$.

[6] S. Engin , N. Sisourat, P. Selles, R. Taïeb and S. Carniato, J. Phys. Chem. A, 2013, 117, 8132.

[7] S. Engin, N. Sisourat, P. Selles, R. Taïeb and S. Carniato, J. Chem. Phys. 2014, 140, 234303.

[8] A. Bonvalet, M. Joffre, J. L. Martin, and A. Migus, Appl. Phys. Lett., 1995, 67, 2907.

[9] J.-P. Likforman, M. Mehendale, D. M. Villeneuve, M. Joffre, and P. B. Corkum, Opt. Lett. 2001, 26, 99.

[10] R. A. Kaindl, M. Wurm, K. Reimann, P. Hamm, A. M. Weiner and M. Woerner, J. Opt. Soc. Am. B, 2000, 17, 2086. 
[11] C. Ventalon, J. M. Fraser, J. P. Likforman, D. M. Villeneuve, P. B. Corkum, and M. Joffre, J. Opt. Soc. Am. B, 2006, 23, 332 .

[12] S. Woutersen, U. Emmerichs, H. J. Bakker, Science, 1997, 278, 658.

[13] M. F. Kropman and H. J. Bakker, J. Chem. Phys., 2001, 115, 8942.

[14] M. F. Kropman, H.-K. Nienhuys, and H. J. Bakker, Phys. Rev. Lett., 2002, 88, 077601.

[15] K. Heyne, N. Huse, E. T. J. Nibbering and T. Elsaesser, J. Phys.: Condens. Matter, 2003, 15, S129.

[16] A. Douhal, F. Lahmani and A. H. Zewail, Chemical physics, 1996, 207, 477.

[17] B.Schiott, B. Br. Iversen, G. K. H. Madsen, F. K. Larsen and T. C. Bruice, PNAS, 1998, 95, 12799.

[18] D. Marx, ChemPhysChem , 2006, 7, 1848.

[19] G. Alagona and C. Ghio, Int. J. Quant. Chem., 2008, 108, 1840.

[20] N.V. Belova, V.V. Sliznev, H. Oberhammer and G.V. Girichev, J. Mol. Struct., 2010, 978, 282.

[21] T. Kaweetirawatt, T. Yamaguchi, T. Higashiyama , M. Sumimoto and K. Hori, J. Phys. Org. Chem., 2012, 25, 1097.

[22] S.H. Bauer and C.F. Wilcox, Chem. Phys. Lett., 1997, 279, 122.

[23] S.F. Tayyari and F. Milani-nejad, Spectrochimica Acta Part A, 2000, 56, 2679.

[24] S. Schlund, E.M. Basilio Janke, K. Weisz and B. Engels, J. Comput. Chem., 2009, 31, 665.

[25] P.I. Nagy, G. Alagona and C. Ghio, J. Chem. Theory Comput., 2007, 3, 1249.

[26] V.V. Sliznev, S.B. Lapshina and G.V. Girichev, J. Struct. Chem., 2006, 47, 220.

[27] J.L. Burdett and M.T. Rogers, J. Am. Chem. Soc., 1964, 86, 2105.

[28] M. Tanaka, T. Shono and K. Shinra, Bull. Chem. Soc. Jpn., 1969, 42, 3190.

[29] A.H. Lowrey, C. George, P. DAntonio and J. Karle, J. Am. Chem. Soc., 1971, 93, 6399.

[30] A.L. Andreassen abd S.H. Bauer, J. Mol. Struct. 1972, 12, 381.

[31] M.M. Folkendt, B.E. Weiss-Lopez, J.P. Chauvel and N.S. True, J. Phys. Chem., 1985, 89, 3347.

[32] K. Iijima, A. Ohnogi and S. Shibata, J. Mol. Struct., 1987, 156, 111.

[33] J.W. Bunting, J.P. Kanter, R. Nelander and Z. Wu, Can. J. Chem., 1995, 73, 1305.

[34] A. Grushow and T.J. Zielinski, J. Chem. Educ., 2002, 79, 707.

[35] R. Srinivasan, J.S. Feenstra, S.T. Park, S. Xu and A.H. Zewail, J. Am. Chem. Soc., 2004, 
126, 2266 .

[36] M. Temprado, M.V. Roux, P. Umnahanant, H. Zhao abd J.S. Chickos, J. Phys. Chem. B, 2005, 109, 12590 .

[37] S. Karabulut and H. Namli, J. Mol. Struct., 2012, 1024, 151.

[38] N.V. Belova, H. Oberhammer, N.H. Trang and G.V. Girichev, J. Org. Chem., 2014, 79, 5412.

[39] G. Alagona, C. Ghio and P. I. Nagy, Phys. Chem. Chem. Phys., 2010, 12, 10173-10188.

[40] A. Schweig, H. Vermeer and U. Weidner, Chem. Phys. Lett., 1974, 26, 229.

[41] H. Jònsson, G. Mills and K. W. Jacobsen, Nudged elastic band method for finding minimum energy paths of transitions in Classical and Quantum Dynamics in Condensed Phase Systems, edited by B. J. Berne, G. Cicotti, and D. F. Coker, World Scientific, 1998.

[42] G. Kresse and J. Hafner, Phys. Rev. B, 1993, 47, 558.

[43] G. Kresse and J. Furthmuller, Phys. Rev. B, 1996, 54, 11169.

[44] D.E. Blöchl, Phys. Rev. B, 1994, 50, 17953.

[45] J.P. Perdew and J. Wang, Phys. Rev. Lett., 1992, 46, 6671.

[46] J. Schirmer, A.B. Trofimov, G. Stelter, J. Chem. Phys., 1998, 109, 4734.

[47] A. B. Trofimov and J. Schirmer, J. Chem. Phys., 2005, 123, 144115.

[48] D. Danovich, WIREs Comput. Mol. Sci., 2011, 1, 377.

[49] M. W. Schmidt, K. K. Baldridge, J. A. Boatz, S. T. Elbert, M. S. Gordon, J. H. Jensen, S. Koseki, N. Matsunaga, K. A. Nguyen, S. J. Su, T. L. Windus, M. Dupuis, J. A. Montgomery, J. Comput. Chem., 1993, 14, 1347.

[50] R. Krishnan, J.S. Binkley, R. Seeger and J.A. Pople, J. Chem. Phys., 1980, 72, 650.

[51] N. S. Hush, M. K. Livett, J. B Peel and G. D. Willett, Aust. J. Chem., 1987, 40, 599.

[52] G. Angonoa, O. Walter, and J. Schirmer, J. Chem. Phys., 1987, 87, 6789.

[53] W.J. Hehre, R. Ditchfield, J.A. Pople, J. Chem. Phys., 1972, 56, 2257.

[54] S.Carniato and P. Millié, J. Chem. Phys., 2002, 116, 3521.

[55] A. D. Becke, J. Chem. Phys., 1993, 98, 5648.

[56] C. Lee, W. Yang, and R. G. Parr, Phys. Rev. B, 1988, 37, 785.

[57] L. Triguero, O. Plashkevych, L. G. M. Pettersson, and H. Agren, J. Electron. Spetrosc. Relat. Phenom., 1999, 104, 195.

[58] D.J. Nesbitt and R.W. Field, J. Phys. Chem., 1996, 100, 12735.

[59] L.S. Cederbaum, W. Domcke, J. Schirmer, W. Von Niessen, Adv. Chem. Phys., 1986, 65, 115. 
[60] R.S. Brown, A. Tse, T. Nakashima and R.C. Haddon, J. Am. Chem. Soc., 1979, 101, 3157.

[61] W.L. Jolly, K.D. Bomben, C.J. Eyermann, At. Data Nucl. Data Tables, 1984, 31, 433.

[62] J.H.S.Q. Perera, D.C. Frost and C.A. McDowell, J. Chem. Phys., 1980, 72, 5151. 
Enolone form

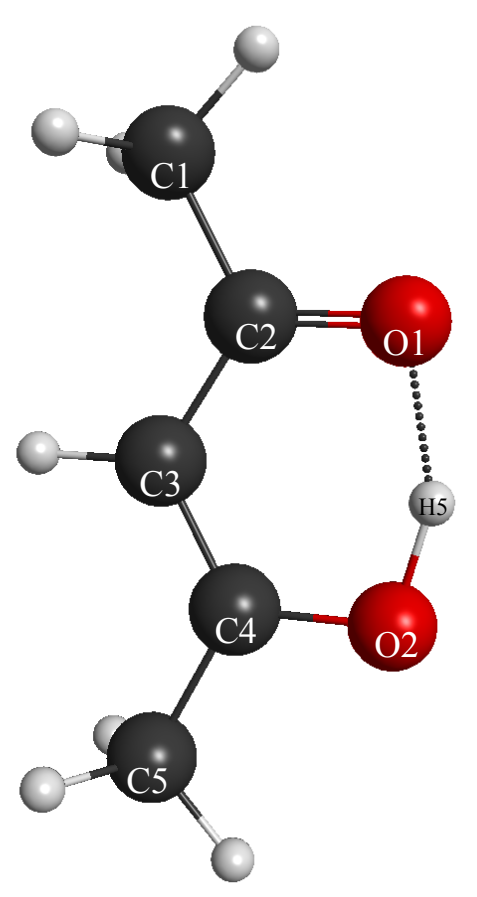

i4 (T.S.)

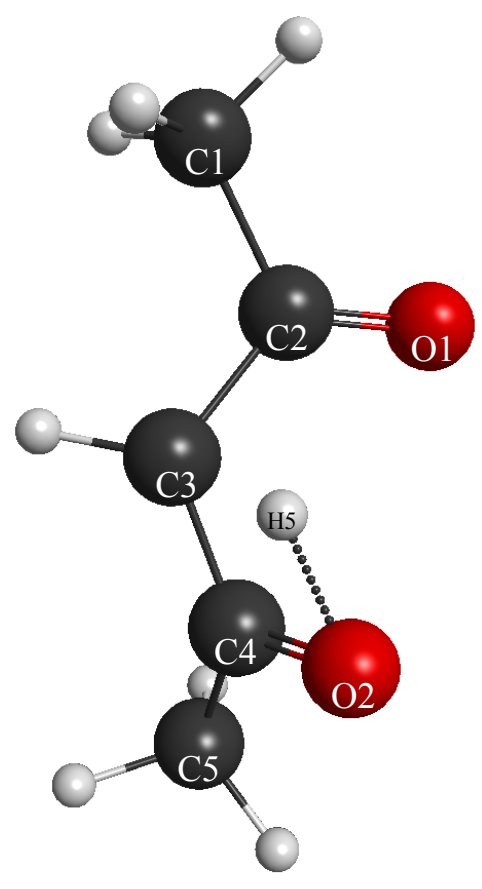

Diketo form

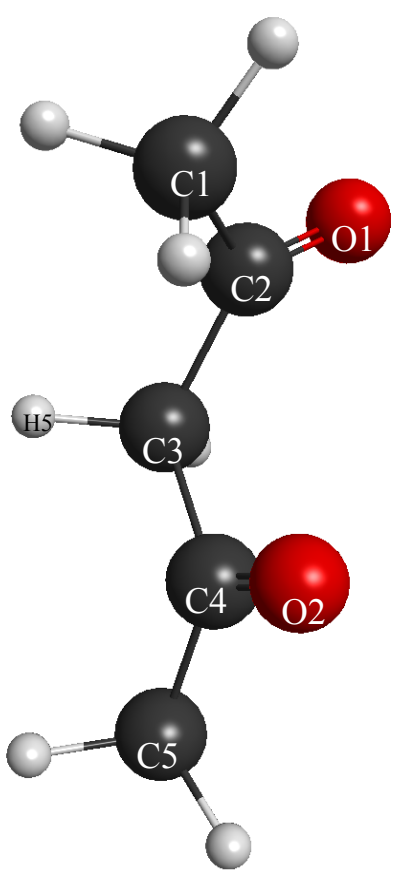

Figure 1. Enolone, diketo and transition-state geometry of the acetylacetone obtained with the NEB technique combined with PWA-GGA 


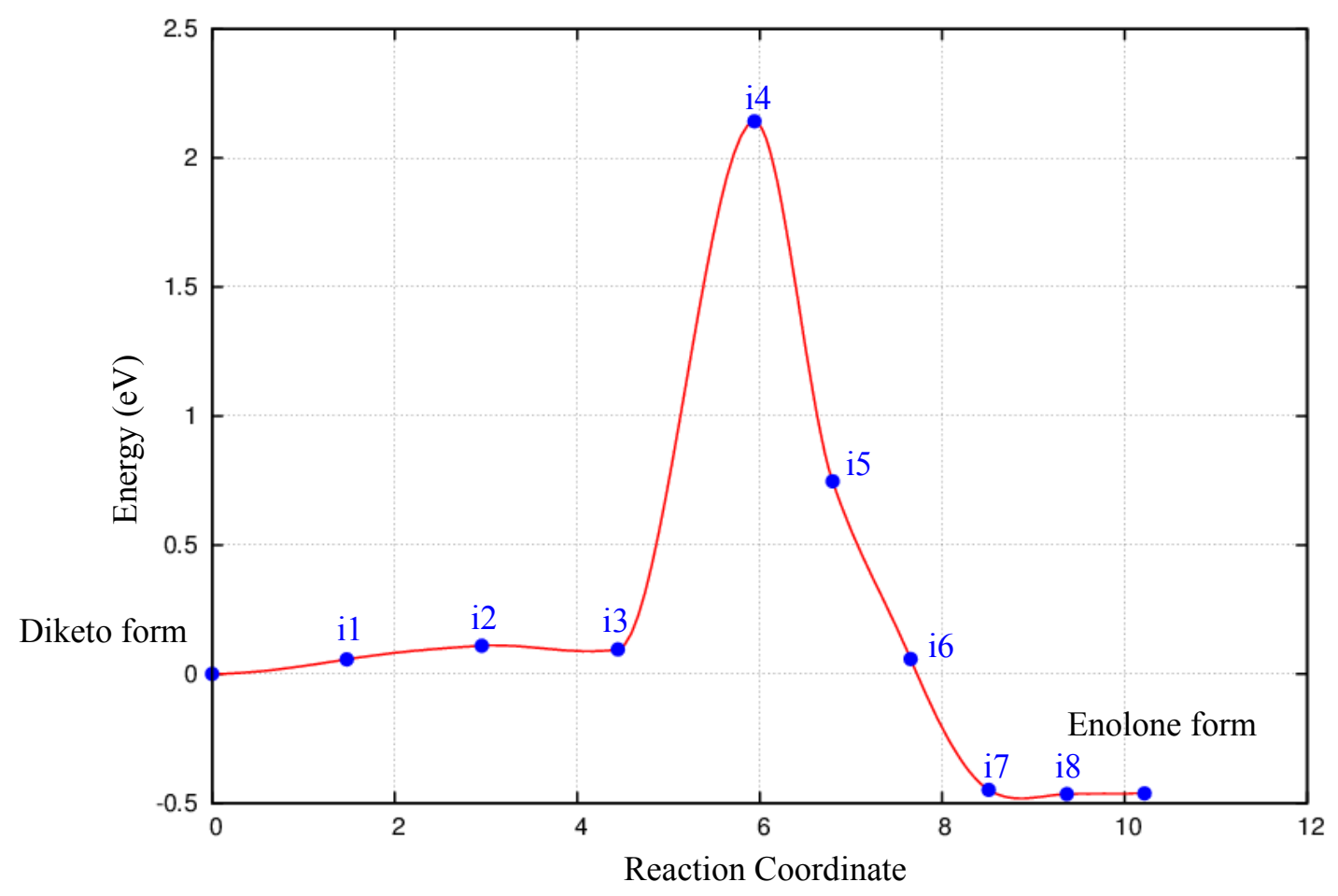

Figure 2. NEB minimum energy path 


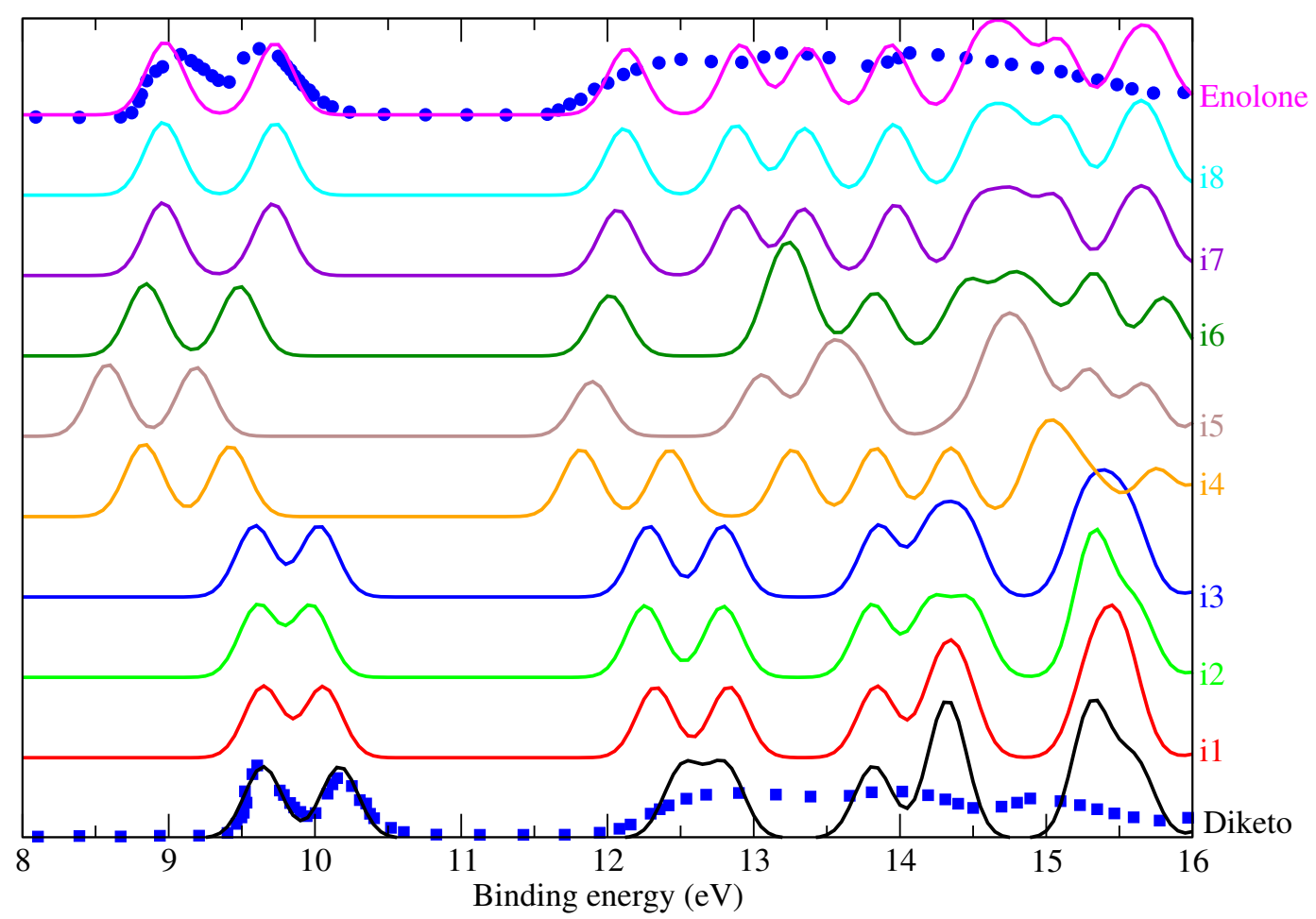

Figure 3. Valence ionization spectra of the acetylacetone in the geometries obtained with the NEB technique. The experimental data for the enolone and diketo forms obtained by Hush et al. ${ }^{51}$ are shown in dots. 


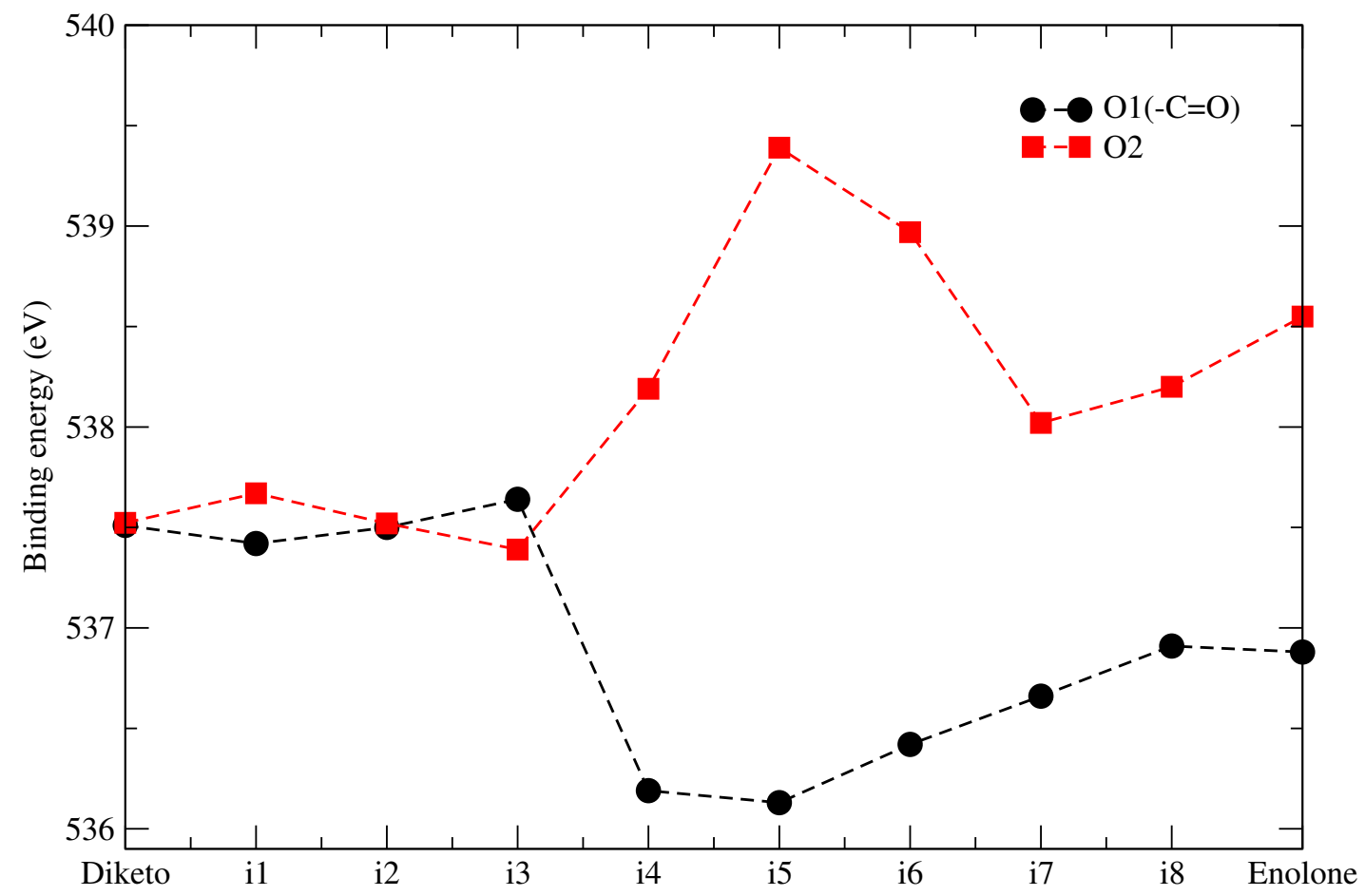

Figure 4. Oxygen core-shell ionization spectra of the acetylacetone in the geometries obtained with the NEB technique. 